\title{
Neferine Enhances Autophagy to Alleviate \\ Cytotoxicity and Apoptosis Induced by CSE in AEC- II through AMPK/mTOR Signaling Pathway
}

\section{Mengmeng Wang}

Shanghai Jiaotong University School of Medicine Xinhua Hospital https://orcid.org/0000-0002-22638369

Haiyang Yu

Shanghai Jiaotong University School of Medicine Xinhua Hospital

\section{Yuqing Sun}

Shanghai Jiaotong University School of Medicine Xinhua Hospital

\section{Pengpeng Cheng}

Shanghai Jiaotong University School of Medicine Xinhua Hospital

\section{Qian Wang}

Shanghai Jiaotong University School of Medicine Xinhua Hospital

Fengfeng Han ( $\sim$ hanfengfeng@xinhuamed.com.cn )

Xinhua Hospital Affiliated To Shanghai Jiaotong University School Of Medicine

Original article

Keywords: CSE, Neferine, Autophagy, AEC-II, AMPK/mTOR

Posted Date: September 9th, 2020

DOI: https://doi.org/10.21203/rs.3.rs-61062/v1

License: (c) (1) This work is licensed under a Creative Commons Attribution 4.0 International License.

Read Full License 
Neferine Enhances Autophagy to Alleviate Cytotoxicity and Apoptosis Induced by CSE in AEC-II through AMPK/mTOR Signaling Pathway

Mengmeng Wang ${ }^{1}{ }^{2}$, Haiyang Yu ${ }^{1}$, Yuqing Sun ${ }^{1}$, Pengpeng Cheng ${ }^{1}$, Qian Wang ${ }^{1}$, and Fengfeng Han ${ }^{1}$

${ }^{1}$ Department of Pulmonary Medicine,Xinhua Hospital Affiliated To Shanghai Jiaotong University School Of Medicine,1653 Kongjiang Road, Yangpu District, Shanghai, China

${ }^{2}$ Department of Pulmonary Medicine, Shanghai Xuhui District Central Hospital, 966 Huaihai Middle Road, Xuhui District, Shanghai, China 


\begin{abstract}
COPD is a clinical common chronic respiratory disease, its incidence case fatality rate is higher, there is currently no cure drugs and methods. In this study, in order to make clear its role in the development of autophagy in COPD, COPD cell model is established.To further explore whether regulating autophagy could have a protective effect to fight against CSE-induced cytotoxicity and apoptosis, we choose neferine as an autophagy inducer. Neferine activated cell autophagy in an vitro CSE-induced COPD cell model and gradually attenuated CSE-induced cell apoptosis. Furthermore, this process happens largely through the AMPK/mTOR signaling pathway. As a autophagic flux inhibitor, chloroquine abolished the prosurvival autophagy effect, and AMPK inhibitor Compound $\mathrm{C}$ blocked neferine-mediated autophagy and then neferine failed to protect COPD cell model from CSE-induced apoptosis. Overall,our findings suggested that neferine possibly has a potentially protective effect in cell damage mechanisms caused by CSE. It hints that neferine has the prospect of turning into a potential therapeutics to cure CSE-induced cytotoxicity and apoptosis and even COPD patients.
\end{abstract}

Key words: CSE; Neferine; Autophagy; AEC-II; AMPK/mTOR 


\section{Introduction}

Chronic obstructive pulmonary disease (COPD) is a clinical common chronic respiratory disease characterized by persistent airflow limitation ${ }^{[1-3]}$.Smoking is the most common risk factor for COPD.Smoking inhalation can cause airway pathophysiological changes and then result in airflow limitation. Nevertheless, the mechanism by which smoking provokes the airflow limitation in COPD patients has not been clearly determined, although inflammation ${ }^{[4]}$, oxidative stress ${ }^{[5]}$, and protease-antiprotease imbalance ${ }^{[6]}$ have all been proposed to contribute to the development of COPD ${ }^{[7]}$. Several studies have proved that autophagy and apoptosis play roles in pathophysiological changes of airflow limitation ${ }^{[8]}$.

Autophagy, a highly conserved autodigestion cellular program, aim to deliver cytoplasmic materials to the lysosome and then maintain cellular homeostasis ${ }^{[9]}$. Many studies have shown autophagy has an essential role in the regulation of intracellular homeostasis and even in the pathogenesis of some chronic diseases ${ }^{[10]}$.Initiation of autophagy and its important functions have sparked heated discussion in respiratory disease ${ }^{[11]}$.Cigarette smoke extract (CSE) was usually used to make COPD cell model ${ }^{[12,13]}$.Alveolar epithelial type-II cells (AEC-II) of the rat is a stem cell of alveolar epithelium, whose functions include proliferation, damage repair, synthesis and secretion of pulmonary surfactant (PS), maintenance of fluid balance inside and outside the alveoli, and involvement in immune regulation. The impact of CSE on AEC-II is worth exploring further. ${ }^{[14,15]}$

Neferine is a major bisbenzylisoquinoline alkaloid extracted from the green embryos of Nelumbo nucifera Gaertn(Fig.4.).Considering its non-toxic natureis , Nelumbo nucifera Gaertn is often used as a traditional Chinese medicine and even used to make soup or tea. It not only is a strong inducer of autophagy ${ }^{[16-19]}$ but also has anti-apoptosis properties ${ }^{[20]}$.Increasing researches begin to focus on the possibly protective role of neferine in disease pathology, such as ROS-mediated oxidative stress and tissue injury ${ }^{[10,20]}$. Furthermore, we hypothesized that neferine may have a protective role in reducing apoptosis in the pathogenesis of COPD.However, the protective mechanisms remain unclear. With these findings and hypotheses, we intended to explore the cytoprotective effect of neferine on CSE-induced cytotoxicity and apoptosis in an in-vitro model.

In this study, by establishing an in-vitro CSE-induced COPD cell model, we aim to confirm that neferine can enhance autophagy and reduce apoptosis without affecting the normal function of cells. Mechanistically, we showed that neferine plays an important role in inducing autophagy through AMPK/mTOR signaling pathway. Taken together, our results report that neferine may alleviate cell damage caused by CSE by enhancing autophagy, which can provide research basis for the future therapeutic application of COPD. 


\section{Materials and Methods}

\subsection{Chemicals and antibodies}

Neferine (98.2\% by HPLC with MW624.77), obtained from Chengdu purechem-standard co., LTD , diluted to concentration $3 \mathrm{mM}$ with DMSO as stock solution. Chloroquine(autologouslysosomal degradation blocker) and Compound C(AMPK inhibitor) were obtained from Sigma-Aldrich.Primary monoclonal antibodies for Beclin-1, p62, LC3, mTOR, p-mTOR, phospho-AMPK, and GAPDH were all purchased from Abcam. The secondary antibodies both for anti-mouse and anti-rabbit were purchased from Cell Signaling Technology. Chemicals and antibodies for testing were prepared freshly.

\subsection{Cell culture and treatment}

Alveolar epithelial type-II cells of the rat(AEC-II) was cultured in $10 \%$ fetal bovine serum( FBS) and DMEM, Ham's F-12 nutrient medium mixture (DMEM/F-12; Invitrogen-Gibco) at $37^{\circ} \mathrm{C}$ under $5 \%$ (vol/vol) $\mathrm{CO}_{2}$. According to each experiment, AEC-II were plated at an appropriate confluence. AEC-II stimulated with CSE was used to make COPD cell model. For stimulation, cells were exposed to the gradient concentrations of neferine, and then pretreated with chloroquine and Compound C. At last, cells were collected for analysis.

\subsection{Preparation of cigarette smoke extract}

Cigarette smoke extract (CSE) was made by a waterpipe three-tube smoking system and prepared from fifiltered cigarettes (CHIENMEN, each cigarette containing $11 \mathrm{mg}$ tar and $0.8 \mathrm{mg}$ nicotine,Daqianmen Group, Inc.,China Hong Kong) as described previously ${ }^{[12,21,22]}$. Briefly, smoke was continuously collected from 2 cigarette and babbled through $20 \mathrm{~mL}$ prewarmed serum-free DMEM/F-12 containing 10\% PBS into a bottle connected to the tee. Each cigarette needed to be fully burned within 2 minute. The $\mathrm{pH}$ of the CSE was adjusted to 7.4. The CSE was then identified as $100 \%$ CSE ,sterilized through a $0.22 \mu \mathrm{M}$ filter (Sartorius Stedim Biotech, Goettingen, Germany), and stored at $-80^{\circ} \mathrm{C}$. CSE diluted to different concentrations in cell culture medium for each experiment.

\subsection{Cell counting kit-8(CCK8)}

Cell proliferation and viability were measured with CCK8(Tongren Company, Japan). AEC-II was seeded at a suitable density in a 96-well plate and incubated until the cells confluent to $60 \%$. Then add different concentrations of CSE or neferine to test its toxic effect on AEC-II. After stimulation for $24 \mathrm{~h}, 10 \mathrm{ml}$ of CCK8 and $100 \mathrm{ml}$ of 
DMEM/F-12 containing 10\% PBS were added to each well, and after incubation at $37^{\circ} \mathrm{C}$ for $1 \mathrm{~h}, 2 \mathrm{~h}, 3 \mathrm{~h}$, and $4 \mathrm{~h}$, the absorbance values were measured by CCK 8 assay after incubation to calculate the cell viability, and the color change was observed.

\subsection{Western blot analysis}

After treatment,cell protein was lysed in RIPA buffer(Beyotime, China) and supplemented with protease and phosphoric acid inhibitors.BCA protein assay kit(Beyotime, China) was used to detect the protein concentration. Different proteins were separated by SDS-PAGE and then transferred to membranes (Millipore, Bedford, MA), which were blocked in $5 \%$ nonfat milk for $2 \mathrm{~h}$ and were incubated at $4{ }^{\circ} \mathrm{C}$ overnight with specific antibodies against GAPDH (Cell Signaling),actin (Cell Signaling),LC3B, p62, mTOR,p-mTOR,AMPK and p-AMPK at a concentration of 1 : 1000 as recommended previously at at $4^{\circ} \mathrm{C}$ overnight. Membranes were incubated for

$2 \mathrm{~h}$ at room temperature with secondary antibodies and then washed three times with TBST. The blots result were scanned by ChemiDocTM MP Imaging System ${ }^{[17]}$.

\subsection{Cellular immunofluorescence assay}

AEC-II was inoculated onto a sterilized coverslip. After cultivation, AEC-II cells were washed 3 times using phosphate buffered saline, and then fixed with $4 \%$ paraformaldehyde for 20 minutes. Cells needed to be ruptured with $0.1 \%$ Triton X-100 (Biosharp), and washed with PBS 3 times, and with 3\% bovine serum albumin. Block for 30 minutes and then incubate with the antibody (LC3II, Servicebio,

GB13124) overnight at $4^{\circ} \mathrm{C}$. Block for 30 minutes and then incubate with the primary antibody Servicebio, GB25303) for 50 minutes. DAPI was used for nuclear staining (blue) at room temperature for 10 minutes. Images of AEC-II were snapped using an upright fluorescence microscope (NIKON ECLIPSE C1, Japan) using a NIKON DS-U3 imaging system.

\subsection{Electron microscopy}

AEC-II were seeded into a 6-well plate and incubated until the cells confluent to $60 \%$ and then incubated with CSE for $24 \mathrm{~h}$ and/or neferine for $2 \mathrm{~h}$. After fixed with $2.5 \%$ glutaraldehyde, AEC-II cells were analyzed by transmission electron microscope (TEM). Next, the cells were sequentially fixed and stained. Last,cells' photos were recorded under an electron microscope (PhilipsCM20) to observing magnetic domain structures.

\subsection{Flow Cytometric Analysis of Apoptosis}

Following the Annexin V/PI method, flow cytometric analysis of apoptosis was 
performed according to the instructions of Annexin V-FITC/PI Apoptosis Detection Kit (BD Pharmingen, CA, USA).AEC-II were incubated in 6-well plates overnight. After CSE/neferine/ chloroquine/Compound $\mathrm{C}$ treatment and digested with trypsin, the cells were recovered and washed 3 times with $\mathrm{PBS}^{[23,24]}$. Then the cells were incubated at $37^{\circ} \mathrm{C}$ for 15 minutes in the dark and stained with a mixture of Annexin V-FITC and PI. After addition of FITC-conjugated Annexin V, the specific fluorescence of AEC-II cells were immediately analyzed by FACS Calibur (BD Biosciences, CA, USA) within 1 hour .

\subsection{Statistics}

All Statistics were performed using SPSS version 18.0 at least in triplicate. The results are counted as the mean(SD) with $P$ value of less than 0.05 as statistically significant. Groups containing multiple comparisons were analyzed by ANOVA with t-test, one-way analysis and Tukey's multiple-comparison test.All experiments were repeated 3 times.

\section{Results}

3.1. Effect of CSE on cell viability and autophagy in AEC-II
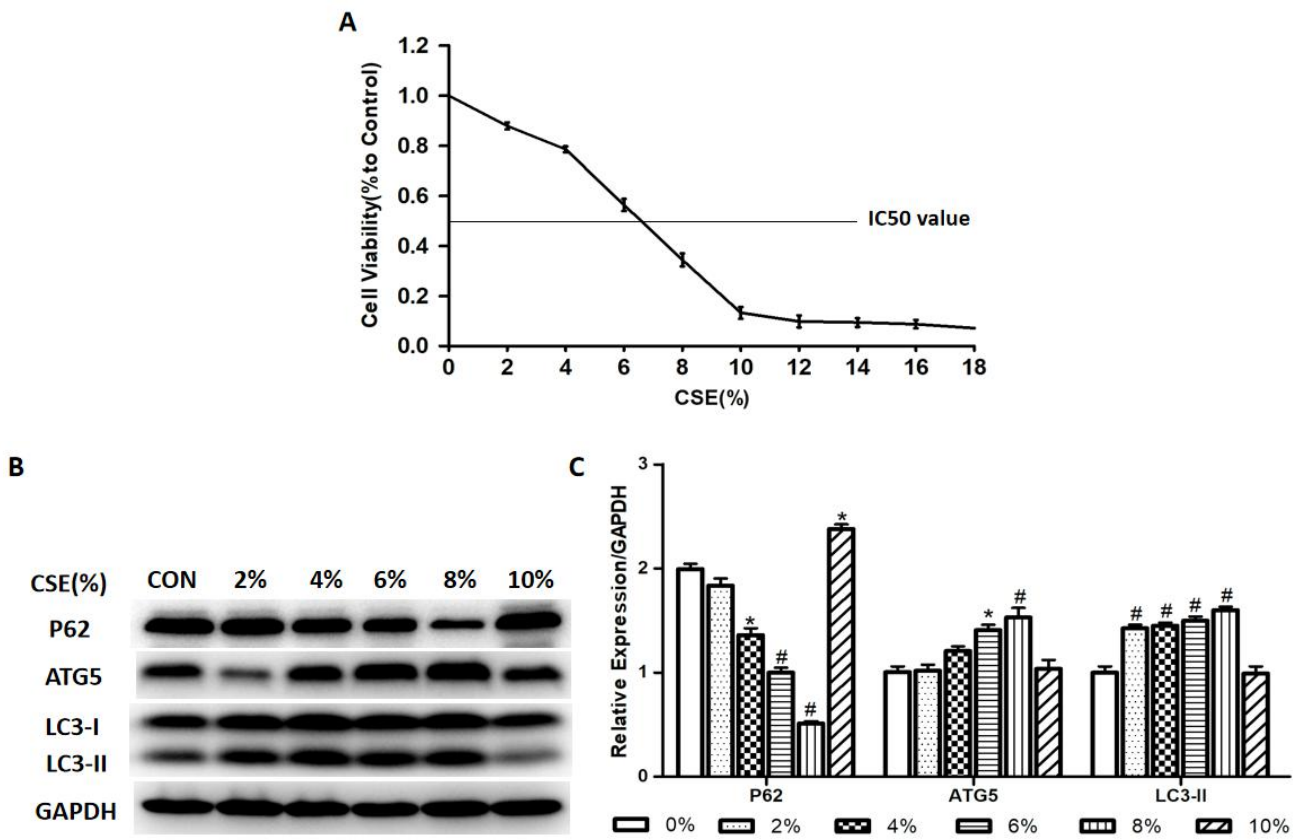
D

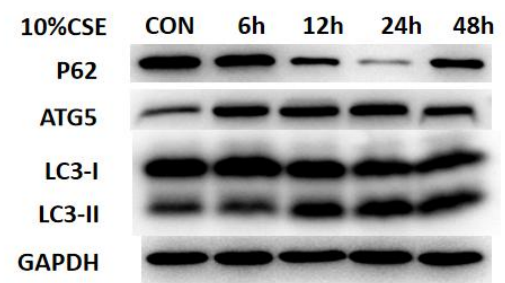

E

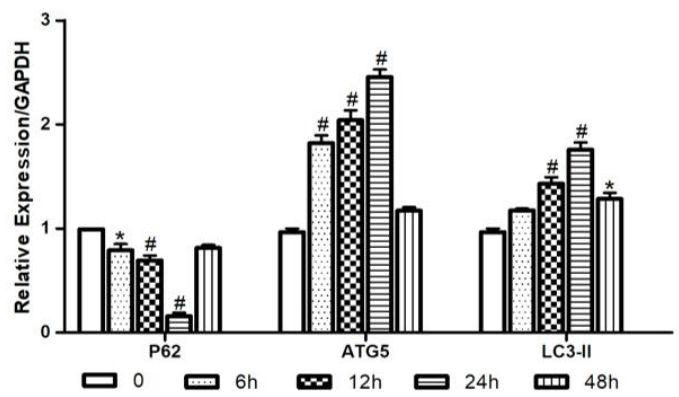

Fig. 1. AEC-II was treated with CSE.(A) CCK8 showing various concentrations of CSE-induced changes in cell viability. Survival curve displayed IC50 value (6.8 \%). (B) GAPDH level is shown as a protein loading control. After $24 \mathrm{~h}$ of various concentrations of CSE (0-10\%) treatment, P62, ATG5 and LC3 protein expression in AEC-II cells were determined by Western blotting analysis. (D) After being subjected to $10 \%$ of CSE for $0-48 \mathrm{~h}$. P62, ATG5 and LC3 protein expression were measured. (C, E) Western blotting analyses results showed average quantitative data and presented as the means \pm SD of at least 3 independent experiments. $* p<0.05$ compared with control; \# $\mathrm{p}<0.01$ compared with control.

Alveolar epithelial type-II cells (AEC-II) of the rat were firstly stimulated by low-to-high concentration gradient of $0 \%-18 \%$ CSE for $24 \mathrm{~h}^{[25,26]}$.CCK-8 assay was used to examine the effect of CSE on the vitality of AEC-II cells(Fig. 1 A). We recorded the cell viability of the CSE group with different concentrations in the form of a percentage expressed as the control group, when the cell viability of the control group was defined as $100 \%$. We observed that the half-inhibitory concentration (IC50) of CSE was $6.8 \%$. The results showed that $2 \%$ CSE had little effect on cell viability of AEC-II, and in the range of $2 \%-10 \%$ CSE cell activity of AEC-II gradually decreased. When the CSE concentration was greater than $10 \%$, the cell viability changed into a platform trend. And then we tested the autophagy of AEC-II caused by in vitro CSE treatment.Western blot analysis confirmed that there was a significant dose- and time-dependent change in the expression levels of P62, ATG5 and LC3II in AEC-II induced by CSE (Fig. 1 B-E). After $24 \mathrm{~h}$ CSE treatment in AEC-II with different concentrations, the results showed that $2 \%-8 \%$ CSE treatment significantly increased the protein expression of Atg5 and LC3-II, while the protein expression of p62 decreased significantly, which is consistent with previous reports ${ }^{[27-29]}$;however, compared with the $8 \%$ CSE group, p62 increased significantly and Atg5 and LC3II decreased obviously when treated with $10 \%$ CSE(Fig. $1 \mathrm{~B}$ and C).After exposure to $10 \% \mathrm{CSE}$ for different different stimulation times, the expression of Atg 5 and LC3II were highest at $24 \mathrm{~h}$, but then decreased for $48 \mathrm{~h}$; the expression of P62 was lowest at $24 \mathrm{~h}$, but then increased for $48 \mathrm{~h}$ (Fig. 1D and E). Therefore, we believed that autophagy is induced during the action of CSE before AEC-II apoptosis or death. It is considered that AEC-II are damaged by the autophagy system under smoke-stimulated conditions at a concentration of $10 \% \mathrm{CSE}$. It was regarded as the best intervention condition that using $10 \% \mathrm{CSE}$ to induce $24 \mathrm{~h}$, and was employed to the subsequent experiments. 
A

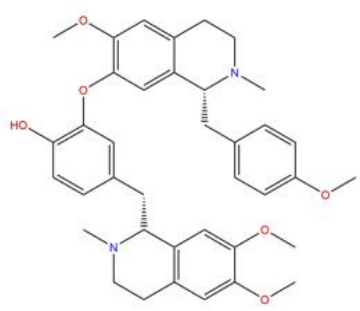

Neferine Chemical Structure

C

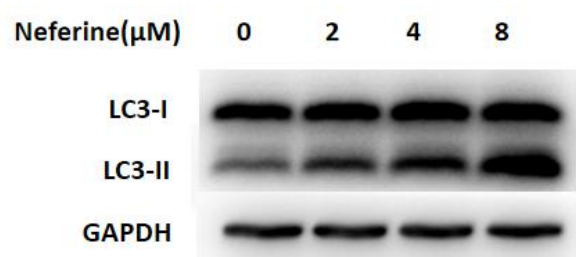

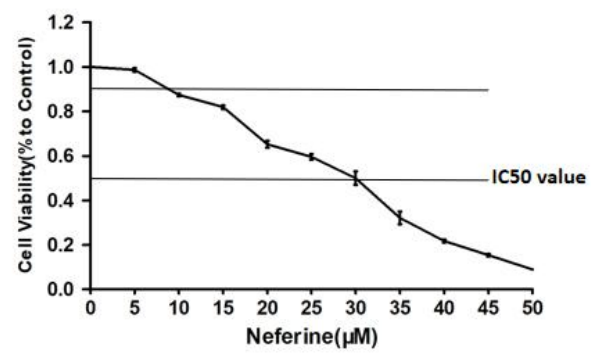

D

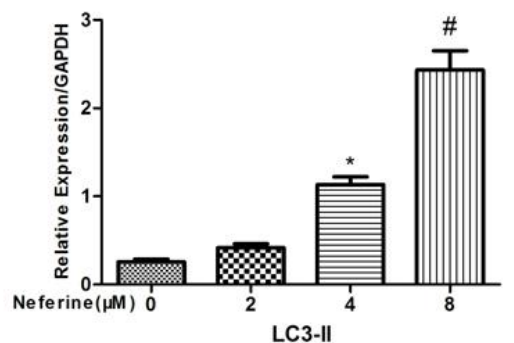

E

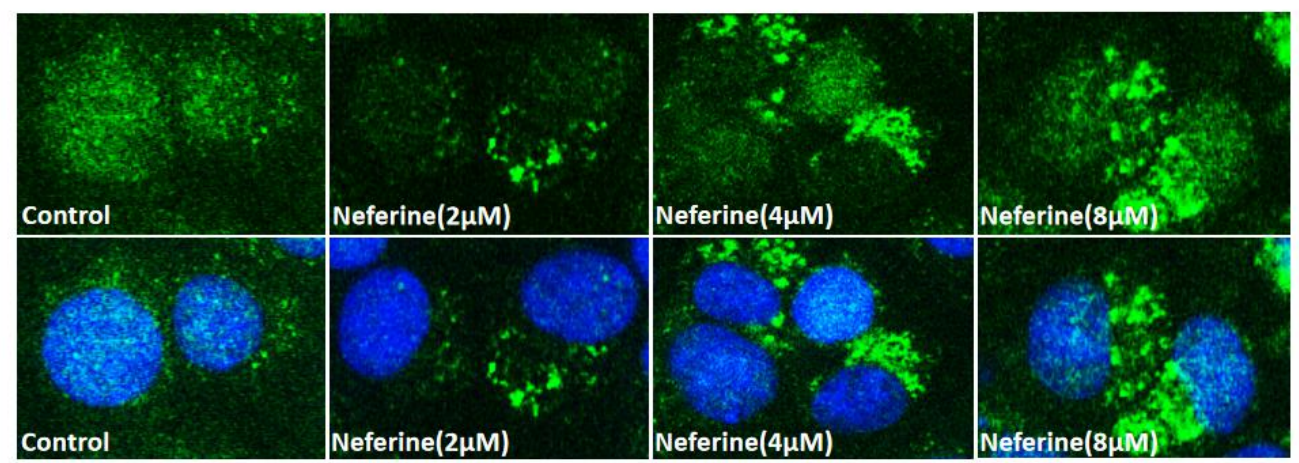

$\mathbf{F}$

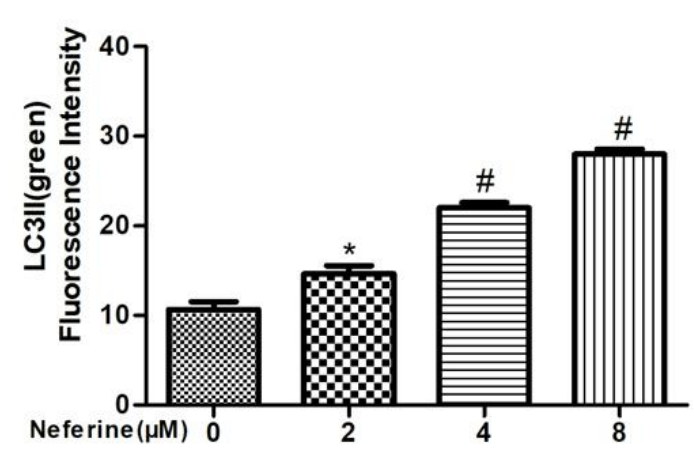

Fig. 2. AEC-II was treated with neferine. (A) The chemical structure of neferine . (B) CCK8 showing various concentrations of neferine-induced changes after $24 \mathrm{~h}$ in cell viability. Survival curve displayed IC50 value (30 $\mu$ M). (C) GAPDH level is shown as a protein loading control. After 24h for different concentrations (2, 4,and $8 \mu$ M), LC3 protein expression in AEC-II cells were determined by Western blotting analysis. (E) Treated with neferine, IF images of AEC-II induced LC3II expression changes (green). Nuclei was labeled with DAPI (blue). $(\mathrm{D}, \mathrm{F})$ Results are presented as the means \pm SD of at least 3 independent experiments. $* \mathrm{p}<0.05$ compared to control; \# $\mathrm{p}<0.01$ compared to control. 
CCK-8 assay was used to examine the role of neferine on the vitality of AEC-II. The viability of AEC-II at other concentrations neferine was expressed as a percentage compared to the control group, under the premise that cell viability of the control group was set as $100 \%$. Result revealed IC50 value was $30 \mu \mathrm{M}$ after $24 \mathrm{~h}$ exposure to neferine (Fig. 2 B). Next, after $24 \mathrm{~h}$ for different concentrations (2, 4, and $8 \mu \mathrm{M}), \mathrm{LC} 3$ protein in AEC-II was induced by neferine (Fig. $2 \mathrm{C}$ and D). Moreover, to visualize LC3II protein, the results of immunofluorescence (IF) staining showed that LC3II protein expression also significantly increased as the concentration of neferine changes. The data further confirmed autophagy induction under the stimulation of neferine (Fig. $2 \mathrm{E}$ and F).

3.3. Effect of neferine on AEC-II survival in CSE by autophagy

A

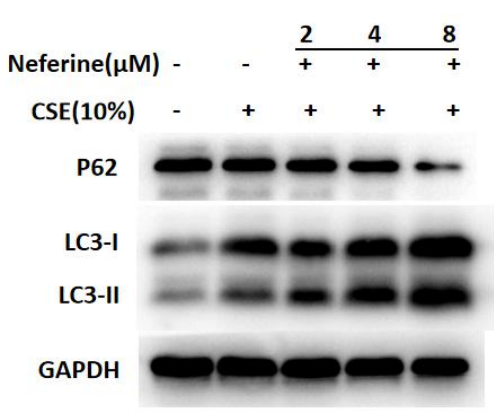

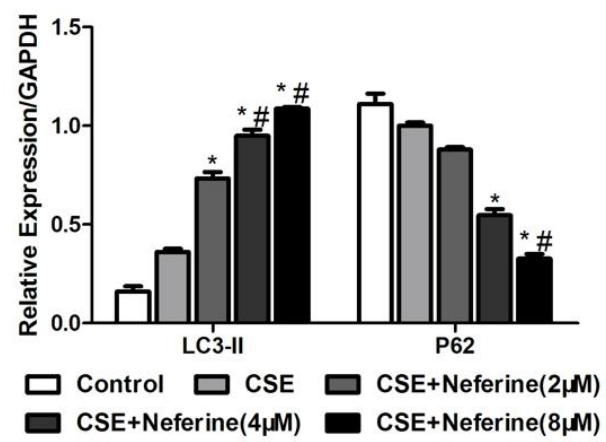

c
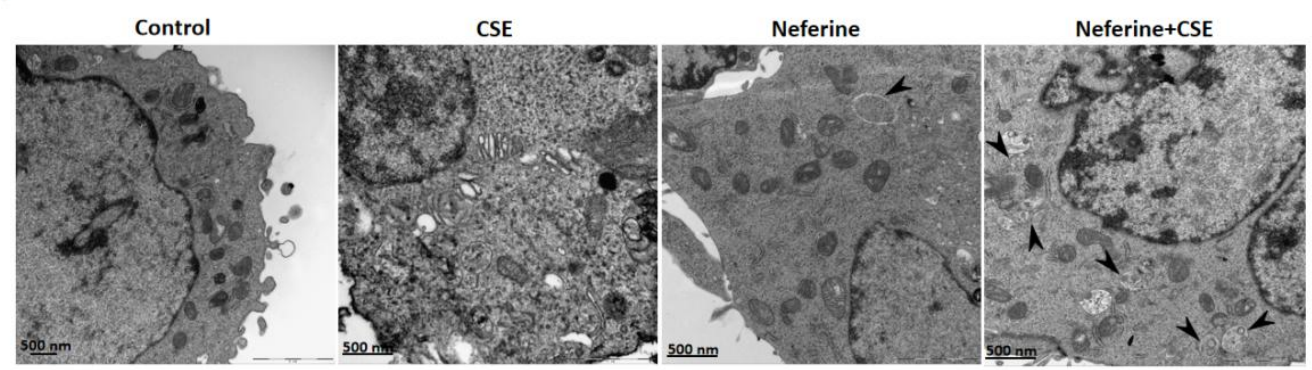

Fig. 3. Effect of neferine on AEC-II survival in CSE by autophagy. (A,B) GAPDH level is shown as a protein loading control. After $2 \mathrm{~h}$ for different concentrations neferine $(0,2,4$,and $8 \mu \mathrm{M})$ with/without $10 \% \mathrm{CSE}$ for $24 \mathrm{~h}$, LC3 and P62 protein expression in AEC-II cells were determined by Western blotting analysis. (C) After $2 \mathrm{~h}$ for neferine at a dose of $8 \mu \mathrm{M}$ with/without $10 \% \mathrm{CSE}$ for $24 \mathrm{~h}$, electron microscope images presented representative autophagosomes or autolysosomes (marked by black arrows;Bar, $500 \mathrm{~nm}$ ). Results are presented as the means \pm $\mathrm{SD}$ of at least 3 independent experiments. ${ }^{*} \mathrm{p}<0.05$ compared to control; $\# \mathrm{p}<0.05$ compared to the CSE group without neferine. 
Some literature pointed out that autophagy may be a major mechanism of airway diseases $^{[11]}$, we assumed that neferine can protect cells by autophagy under certain conditions ${ }^{[30]}$. To confirm this conjecture, we examined the protein expression of LC3 and P62, which are closely involved in the autophagy-related ubiquitination reaction mechanism through $\mathrm{LC}^{[31]}$. Neferine resulted in increased expression of LC3II and decreased expression of $\mathrm{p} 62$ at $8 \mu \mathrm{M}$. This change shows that AEC-II treated with $8 \mu$ $\mathrm{M}$ neferine had stronger autophagy ability. We further made clear that autophagy caused by neferine was found in AEC-II under electron microscope. After $2 \mathrm{~h}$ for neferine at $8 \mu \mathrm{M}$ with/without $10 \% \mathrm{CSE}$ for $24 \mathrm{~h}$, the representative electron microscope images presented more autophagosomes or autolysosomes were identified in AEC-II treated with neferine. In summary, we found that neferine could effectively enhance autophagy activation in AEC-II. Then we chose $8 \mu \mathrm{M}$ as the therapeutic concentration of neferine in the following experiments.

3.4. Autophagy inhibitor attenuated neferine's effect in protecting AEC-II against CSE
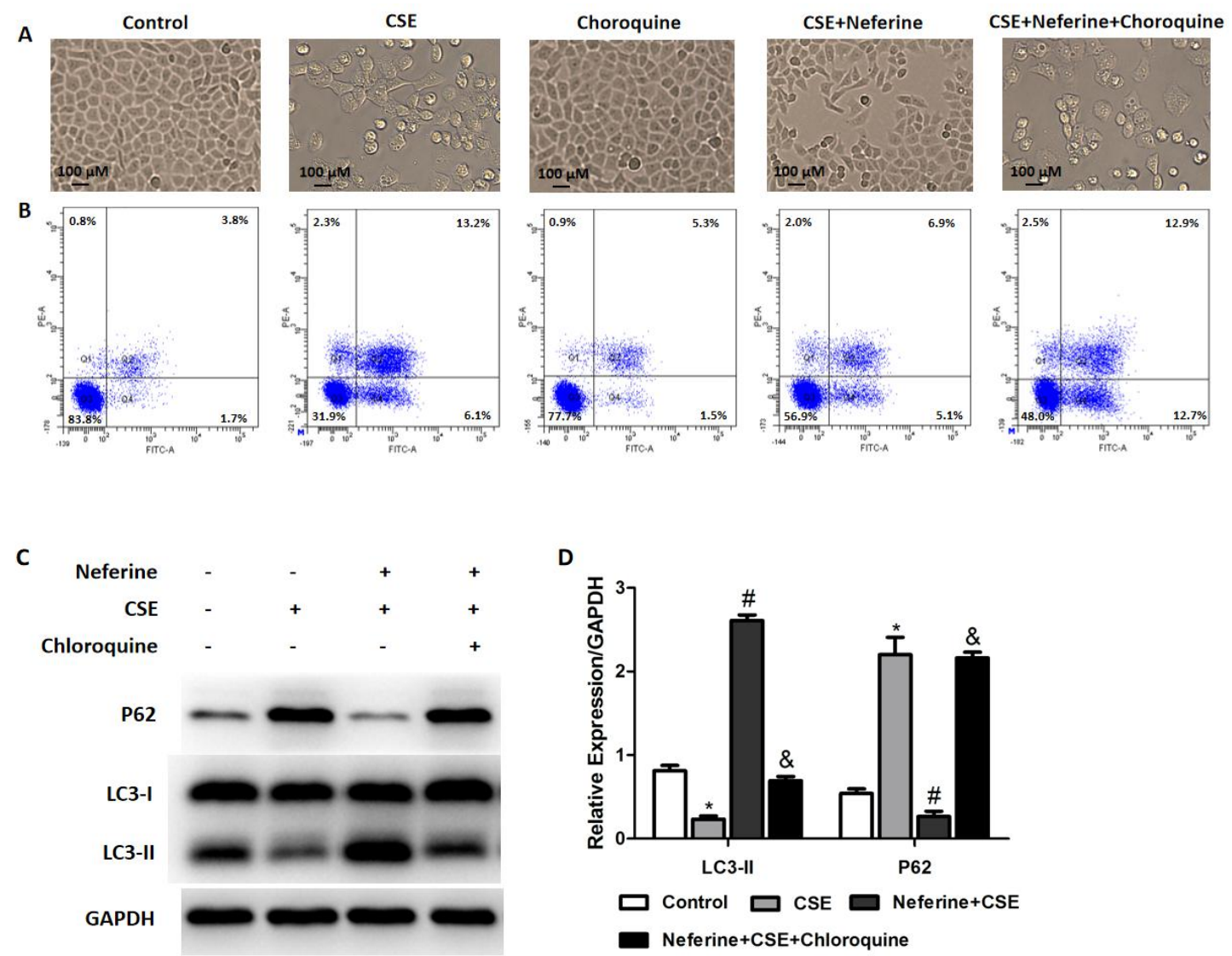

Fig. 4. Autophagy inhibitor attenuated neferine's effect in protecting AEC-II against CSE. AEC-II were pretreated 
for $2 \mathrm{~h}$ with neferine $(8 \mu \mathrm{M})$, or neferine $(8 \mu \mathrm{M})$ together with chloroquine $(20 \mu \mathrm{M})$, followed by treatment with $10 \% \mathrm{CSE}$ for $24 \mathrm{~h}$. (A) Phase-contrast microscopy images presented cells viability (Bar, $100 \mu \mathrm{m}$ ). (B) AEC-II was analyzed by flow cytometric analysis of apoptosis. (C,D) GAPDH level is shown as a protein loading control. LC3 and P62 protein expression in AEC-II were determined by Western blotting analysis.Results are presented as the means \pm SD of at least 3 independent experiments. ${ }^{*} \mathrm{p}<0.05$ compared to control; $\# \mathrm{p}<0.05$ compared to the CSE group; \& $\mathrm{p}<0.05$ compared to the CSE and neferine group.

To further investigate whether neferine can alleviate cytotoxicity and apoptosis of AEC-II induced by CSE via enhance autophagy, chloroquine was used to confirm whether upstream autophagy is activated after stimulation by blocking autochromosomal degradation ${ }^{[8,17,32,33]}$. As we have observed, both morphological observation and flow cytometry analysis showed that chloroquine had no significant effect on cell death and apoptosis, but it could significantly prevent neferine from reducing the protective effect of CSE-induced cell death and apoptosis(Fig. 4A and B). Moreover, western blotting further pointed that the expression of LC3II induced by CSE combined with neferine was higher than CSE alone. However, a decrease in LC3II expression was found in cells treated with CSE, neferine and chloroquine. This indicates that neferine mediated anti-apoptotic protection through autophagy(Fig. 4C and D). The data suggested that neferine significantly induced autophagy. At the same time we detected the protein expression level of another autophagy marker, P62. The expression of P62 induced by CSE combined with neferine was lower than CSE alone. Simultaneously, the increased expression level of P62 was found in cells treated with CSE, neferine, and chloroquine. Collectively, the results indicate that neferine have a cytoprotective effect on AEC-II against CSE-induced death and apoptosis by enhancing autophagy.

3.5. Neferine protected AEC-II against CSE via the AMPK/mTOR pathway
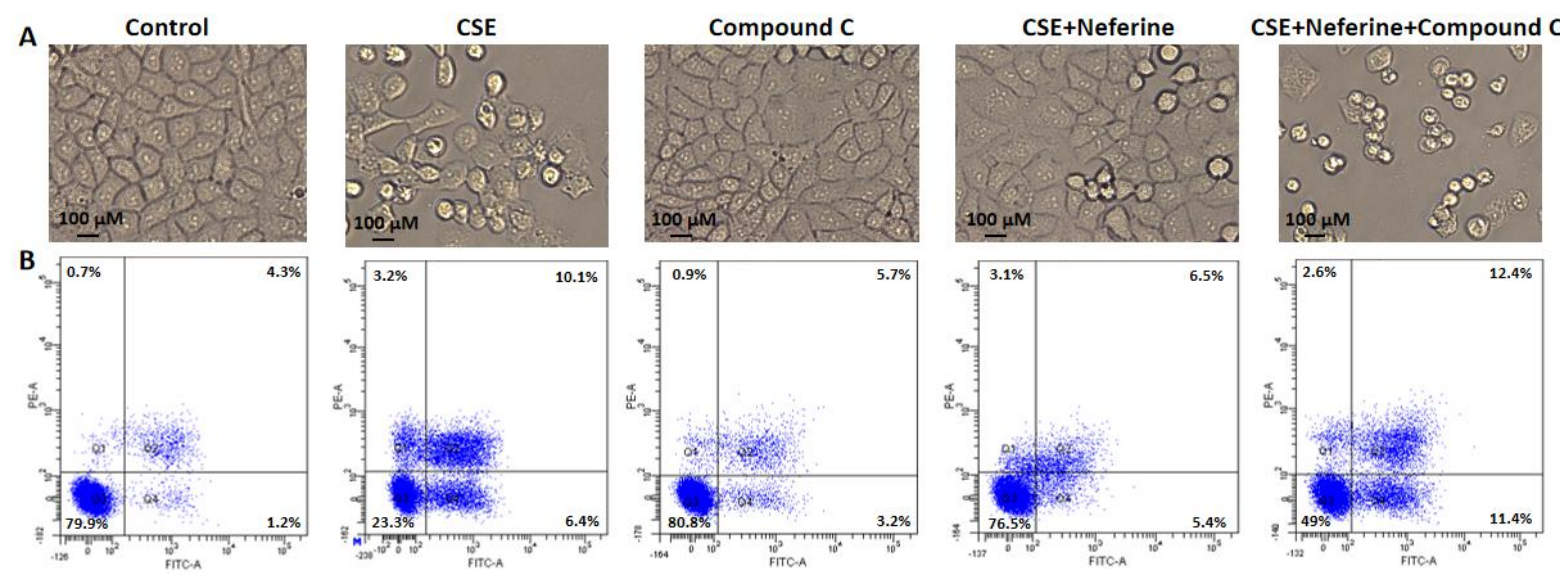


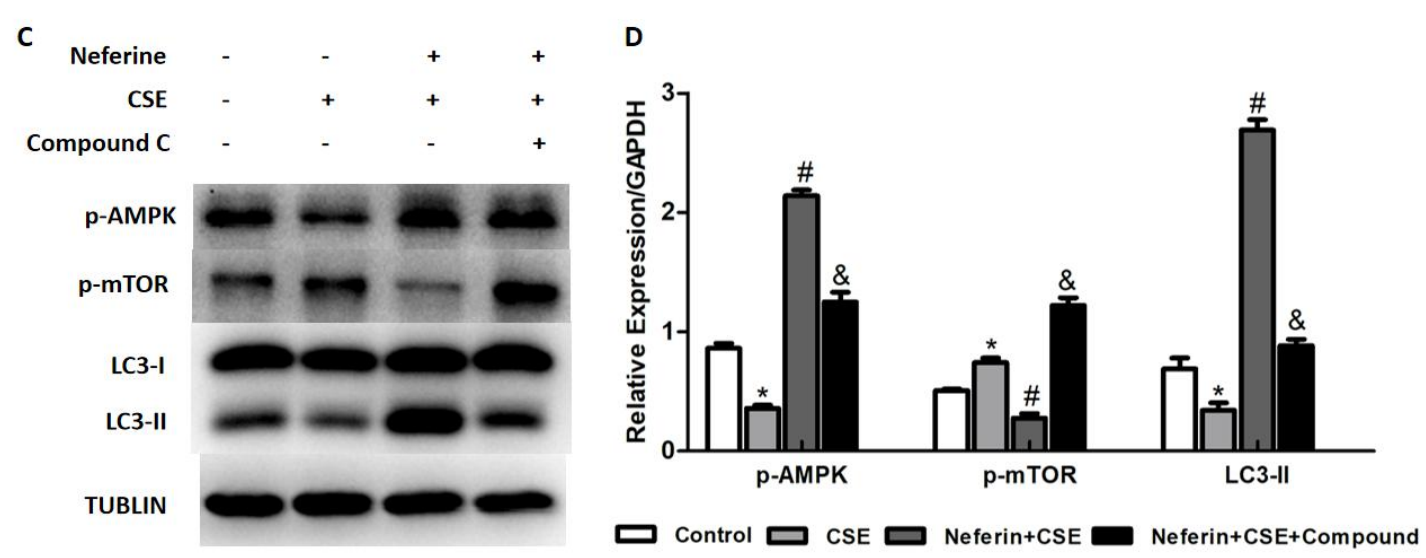

Fig. 5. Neferine protected AEC-II against CSE via the AMPK/mTOR pathway. AEC-II were pretreated for $2 \mathrm{~h}$ with neferine $(8 \mu \mathrm{M})$, or neferine $(8 \mu \mathrm{M})$ together with Compound $\mathrm{C}(10 \mu \mathrm{M})$, followed by treatment with $10 \% \mathrm{CSE}$ for 24h. (A) Phase-contrast microscopy images presented cells viability (Bar, $100 \mu \mathrm{m}$ ). (B) AEC-II was analyzed by flow cytometric analysis of apoptosis. (C,D) GAPDH level is shown as a protein loading control. LC3, p-AMPK, and p-mTOR protein expression in AEC-II were determined by Western blotting analysis.Results are presented as the means \pm SD of at least 3 independent experiments. $* \mathrm{p}<0.05$ compared to control; \# $\mathrm{p}<0.05$ compared to the CSE group; \& $\mathrm{p}<0.05$ compared to the CSE and neferine group.

AMPK / mTOR signaling is the iconic upstream autophagy regulation pathway, and was observed to study neferine by regulating autophagy when AEC-II was stimulated by $\mathrm{CSE}^{[30]}$. Optical microscope observation and flow cytometry analysis statistics (Fig. 5A and 5B) showed that treatment with Compound $\mathrm{C}$ alone had no significant effect on cell death and apoptosis, and Compound $\mathrm{C}$ prevented neferine from protecting AEC-II from CSE. To further explore the potential mechanism, as shown in Fig. 5C and D, we explored the effect of AMPK / mTOR and analyzed the role of Compound $\mathrm{C}$, which was used as a AMPK inhibitor to confirm whether upstream pathway is activated in neferine-induced autophagy ${ }^{[34]}$. Decreased LC3II and p-AMPK expression and increased $\mathrm{p}-\mathrm{mTOR}$ expression revealed that compound $\mathrm{C}$ significantly inhibited autophagy and lost the role of neferine to protect AEC-II from CSE. In summary, these data indicated that neferine induced autophagy by activating AMPK, thereby acting as a protective mechanism against CSE in AEC-II from the apoptosis and death.

\section{Discussion}

In this study, we verified that neferine alleviate CSE-induced AEC-II apoptosis and death by inducting autophagy. We further discovered that the occurrence of this process is closely related to the AMPK / mTOR signaling pathway.

Smoking is one of the most common risk factors for chronic obstructive pulmonary disease. Cigarette smoke contains more than 4,000 kinds of harmful substances, including tar, carbon monoxide, nicotine, benzopyrene, nicotine, nitrogen oxides, 
hydrogen cyanide, acrolein, etc. These substances induce oxidative stress, cytotoxicity, apoptotic,activate corresponding changes in various cells in the lung ${ }^{[19,20]}$. It is common that exposure to CSE is used to explore the mechanism and pathogenesis of COPD in vivo or in vitro. CSE can cause autophagy enhancement ${ }^{[26,27,29,35-37]}$. CSE can cause apoptosis ${ }^{[38-41]}$. We also found that CSE induces autophagy in a time- and dose-dependent manner $(0-24 \mathrm{~h}, 0-8 \%)$. However, when the concentration of CSE is too large, cellular activity is clearly at a low level and autophagy is weakened.We considered that AEC-II are damaged by the autophagy system under smoke-stimulated conditions at an excessive CSE concentration. And CSE does cause apoptosis in AEC-II cells in our experiment. Inhibition of autophagy maybe the pathogenesis of CODP.

Neferine, the major bisbenzylisoquinoline alkaloids from lotus embryos, possesses a variety of pharmacological activities. Previous reports have indicated that neferine has an anti-apoptotic effect. Baskaran et al reported that neferine can block the ROS / Akt / NF-kB pathway to inhibit high glucose-induced apoptosis in endothelial cells ${ }^{[30]}$. In our research, we have explored that neferine could indeed induce enhanced autophagy and neferine pretreatment obviously attenuated CSE-triggered apoptosis. Autophagy process is a dynamic equilibrium process, including initiation, extension, maturation and degradation. The autophagy-associated protein Atg5 is involved in the formation of Atg5 related complex and some ubiquitination protein processing modification processes , which plays a crucial impact on binding to Atg12 and LC3 modification.

In addition to being a marker of autophagy activation via linking LC3 to ubiquitinated proteins, P62 protein can also be an important regulator of apoptosis ${ }^{[36]}$.Neferine treatment enhanced autophagy process, while chloroquine as an autophagy flux inhibitor significantly weakens the protective effect of neferine on CSE-triggerd apoptosis and death, suggesting that neferine induces protection from CSE-induced apoptosis by autophagy.

mTOR, as a conserved serine/threonine protein kinase, is the confluence of upstream pathways including AMPK / mTOR that regulate cell growth, reproduction, movement, survival and autophagy. Studies have confirmed that AMPK regulated mTOR signaling pathway is closely related to intracellular energy balance ${ }^{[38-40,42]}$. Under glucose starvation conditions, AMPK was shown to activate autophagy by directly stimulating the mammalian autophagy promoter kinase Ulk1 ${ }^{[43]}$. Neferine has been shown to have the ability to protect cells from apoptosis due to hypoxia through the Akt / mTOR signaling pathway ${ }^{[44]}$. In the Huntington's disease model, neferine can induce mTOR/AMPK-mediated autophagy ${ }^{[19]}$. Our study also pointed out that neferine mediates autophagy through the AMPK/mTOR signaling pathway. These results indicated to one point: neferine induces autophagy by activating the AMPK / mTOR signaling pathway to prevent CSE-induced AEC-II cytotoxicity and apoptosis.

In conclusion, neferine-induced autophagy may play a cytoprotection role in COPD 
caused by smoking. This may be a promising approach to reduce CSE-induced cytotoxicity and apoptosis and suggested targeting AMPK/mTOR pathway-mediated autophagy as a new treatment strategy for COPD; however, the association between autophagy activity and lung protective mechanisms must be illustrated by further researches. 


\section{Declarations}

Ethics approval and consent to participate

Not applicable.

Consent for publication

All study participants provided informed consent.

Availability of data and material

The datasets used or analysed during the current study are available from the corresponding author on reasonable request.

Competing interests

All authors declared that they have no competing interests. They scanned and approved the final version of manuscript.

Funding

Not applicable.

Authors' contributions

F.H. was corresponding author and supervised the entire research. M.W. was first author and designed the research, interpreted results of experiments, analyzed the data, and drafted the manuscript. M.W., H.Y. and F.H. revised manuscript. Y.S., P.C., and Q.W. involved in performing the cell experiments.

Acknowledgements

We show our thanks to Xinhua Hospital Affiliated To Shanghai Jiaotong University School Of Medicine for providing us with all the facilities for conducting research.

Authors' information (optional)

M.W., Mengmeng Wang, 966 Huaihai Middle Road, Xuhui District, Shanghai, China, icemaggie@foxmail.com.

H.Y., Haiyang Yu, 1653 Kongjiang Road, Yangpu District, Shanghai, China, haiyangdoctor@126.com.

Y.S., Yuqing Sun, 1653 Kongjiang Road, Yangpu District, Shanghai, China, 1260949935@qq.com.

P.C., Pengpeng Cheng, 1653 Kongjiang Road, Yangpu District, Shanghai, China, 626494014@qq.com.

Q.W., Qian Wang, 1653 Kongjiang Road, Yangpu District, Shanghai, China, 626494014@qq.com.

F.H., Fengfeng Han, 1653 Kongjiang Road, Yangpu District, Shanghai, China, hanfengfeng@xinhuamed.com.cn. 


\section{References}

1. Mirza S, Clay RD, Koslow MA, et al: COPD Guidelines: A Review of the 2018 GOLD Report. Mayo Clin Proc 93:1488-1502, 2018

2. Prinzi G, Santoro A, Lamonaca $P$, et al: Cognitive Impairment in Chronic Obstructive Pulmonary Disease (COPD): Possible Utility of Marine Bioactive Compounds. Mar Drugs 16, 2018

3. Vollenweider DJ, Frei A, Steurer-Stey CA, et al: Antibiotics for exacerbations of chronic obstructive pulmonary disease. Cochrane Database Syst Rev 10:Cd010257, 2018

4. Lomas DA: Does Protease-Antiprotease Imbalance Explain Chronic Obstructive Pulmonary Disease? Ann Am Thorac Soc 13 Suppl 2:S130-7, 2016

5. Bodas M, Silverberg D, Walworth K, et al: Augmentation of S-Nitrosoglutathione Controls Cigarette Smoke-Induced Inflammatory-Oxidative Stress and Chronic Obstructive Pulmonary Disease-Emphysema Pathogenesis by Restoring Cystic Fibrosis Transmembrane Conductance Regulator Function. Antioxid Redox Signal 27:433-451, 2017

6. Aghasafari P, George U, Pidaparti R: A review of inflammatory mechanism in airway diseases. Inflamm Res 68:59-74, 2019

7. Tse HN, Tseng CZ: Update on the pathological processes, molecular biology, and clinical utility of $\mathrm{N}$-acetylcysteine in chronic obstructive pulmonary disease. Int J Chron Obstruct Pulmon Dis 9:825-36, 2014

8. Mizumura K, Maruoka S, Shimizu T, et al: Autophagy, selective autophagy, and necroptosis in COPD. Int J Chron Obstruct Pulmon Dis 13:3165-3172, 2018

9. Qian M, Fang X, Wang X: Autophagy and inflammation. Clin Transl Med 6:24, 2017

10. Ohsumi Y: Historical landmarks of autophagy research. Cell Res 24:9-23, 2014

11. Zeki AA, Yeganeh B, Kenyon NJ, et al: Autophagy in airway diseases: a new frontier in human asthma? Allergy 71:5-14, 2016

12. Ferraro $M$, Di Vincenzo $S$, Dino $P$, et al: Budesonide, Aclidinium and Formoterol in combination limit inflammaging processes in bronchial epithelial cells exposed to cigarette smoke. Exp Gerontol 118:78-87, 2019

13. Wang $M$, Zhang $Y, X u M$, et al: Roles of TRPA1 and TRPV1 in cigarette smoke -induced airway epithelial cell injury model. Free Radic Biol Med 134:229-238, 2019

14. Liu G, Chen T, Chen B: [Effect of heme oxygenase-1 on the apoptosis of type II alveolar epithelial cells in rats with hyperoxia-induced acute lung injury]. Zhonghua Wei Zhong Bing Ji Jiu Yi Xue 30:1001-1005, 2018

15. Xu BY, Li YL, Luan B, et al: MiR-26a protects type II alveolar epithelial cells against mitochondrial apoptosis. Eur Rev Med Pharmacol Sci 22:486-491, 2018

16. Marthandam Asokan S, Mariappan R, Muthusamy S, et al: Pharmacological benefits of neferine - A comprehensive review. Life Sci 199:60-70, 2018

17. Kalai Selvi S, Vinoth A, Varadharajan T, et al: Neferine augments therapeutic efficacy of cisplatin through ROS- mediated non-canonical autophagy in human lung adenocarcinoma (A549 cells). Food Chem Toxicol 103:28-40, 2017

18. Poornima P, Weng CF, Padma VV: Neferine from Nelumbo nucifera induces autophagy through the inhibition of PI3K/Akt/mTOR pathway and ROS hyper generation in A549 cells. Food Chem 141:3598-605, 2013

19. Wong VK, Wu AG, Wang JR, et al: Neferine attenuates the protein level and toxicity of mutant huntingtin in PC-12 cells via induction of autophagy. Molecules 20:3496-514, 2015 
20. Guan G, Han H, Yang $Y$, et al: Neferine prevented hyperglycemia-induced endothelial cell apoptosis through suppressing ROS/Akt/NF-kappaB signal. Endocrine 47:764-71, 2014

21. Li Y, Yu G, Yuan S, et al: 14,15-Epoxyeicosatrienoic acid suppresses cigarette smoke condensate-induced inflammation in lung epithelial cells by inhibiting autophagy. Am J Physiol Lung Cell Mol Physiol 311:L970-I980, 2016

22. Kode A, Rajendrasozhan S, Caito $S$, et al: Resveratrol induces glutathione synthesis by activation of $\mathrm{Nrf} 2$ and protects against cigarette smoke-mediated oxidative stress in human lung epithelial cells. Am J Physiol Lung Cell Mol Physiol 294:L478-88, 2008

23. Li H, Tang $Y$, Wen $L$, et al: Neferine reduces cisplatin-induced nephrotoxicity by enhancing autophagy via the AMPK/mTOR signaling pathway. Biochem Biophys Res Commun 484:694-701, 2017

24. Pham DC, Chang YC, Lin SR, et al: FAK and S6K1 Inhibitor, Neferine, Dually Induces Autophagy and Apoptosis in Human Neuroblastoma Cells. Molecules 23, 2018

25. Bodas M, Vij N: Adapting Proteostasis and Autophagy for Controlling the Pathogenesis of Cystic Fibrosis Lung Disease. Front Pharmacol 10:20, 2019

26. Chen G, Zhou M, Chen L, et al: Cigarette Smoke Disturbs the Survival of CD8+ Tc/Tregs Partially through Muscarinic Receptors-Dependent Mechanisms in Chronic Obstructive Pulmonary Disease. PLoS One 11:e0147232, 2016

27. Zhou JS, Zhao $\mathrm{Y}$, Zhou HB, et al: Autophagy plays an essential role in cigarette smoke-induced expression of MUC5AC in airway epithelium. Am J Physiol Lung Cell Mol Physiol 310:L1042-52, 2016

28. Chen $\mathrm{ZH}$, Lam HC, Jin $\mathrm{Y}$, et al: Autophagy protein microtubule-associated protein 1 light chain-3B (LC3B) activates extrinsic apoptosis during cigarette smoke-induced emphysema. Proc Natl Acad Sci U S A 107:18880-5, 2010

29. Hwang JW, Chung S, Sundar IK, et al: Cigarette smoke-induced autophagy is regulated by SIRT1-PARP-1-dependent mechanism: implication in pathogenesis of COPD. Arch Biochem Biophys 500:203-9, 2010

30. Baskaran R, Poornima P, Huang CY, et al: Neferine prevents NF-kappaB translocation and protects muscle cells from oxidative stress and apoptosis induced by hypoxia. Biofactors 42:407-17, 2016

31. Klionsky DJ, Abdelmohsen K, Abe A, et al: Guidelines for the use and interpretation of assays for monitoring autophagy (3rd edition). Autophagy 12:1-222, 2016

32. Jiang $M$, Wei $Q$, Dong $G$, et al: Autophagy in proximal tubules protects against acute kidney injury. Kidney Int 82:1271-83, 2012

33. Xu T, Singh D, Liu J, et al: Neferine, is not inducer but blocker for macroautophagic flux targeting on lysosome malfunction. Biochem Biophys Res Commun 495:1516-1521, 2018

34. Zhang Z, Cheng X, Yue L, et al: Molecular pathogenesis in chronic obstructive pulmonary disease and therapeutic potential by targeting AMP-activated protein kinase. J Cell Physiol 233:1999-2006, 2018

35. Chen ZH, Kim HP, Sciurba FC, et al: Egr-1 regulates autophagy in cigarette smoke-induced chronic obstructive pulmonary disease. PLoS One 3:e3316, 2008

36. Sanchez-Martin P, Komatsu M: p62/SQSTM1 - steering the cell through health and disease. J Cell Sci 131, 2018

37. Vij N, Chandramani-Shivalingappa P, Van Westphal C, et al: Cigarette smoke-induced autophagy impairment accelerates lung aging, COPD-emphysema exacerbations and pathogenesis. 
Am J Physiol Cell Physiol 314:C73-c87, 2018

38. Xu J, Ji J, Yan XH: Cross-talk between AMPK and mTOR in regulating energy balance. Crit Rev Food Sci Nutr 52:373-81, 2012

39. Zhong J, Xie J, Xiao J, et al: Inhibition of PDE4 by FCPR16 induces AMPK-dependent autophagy and confers neuroprotection in SH-SY5Y cells and neurons exposed to MPP(+)-induced oxidative insult. Free Radic Biol Med, 2019

40. Shi C, Xue W, Han B, et al: Acetaminophen aggravates fat accumulation in NAFLD by inhibiting autophagy via the AMPK/mTOR pathway. Eur J Pharmacol 850:15-22, 2019

41. Tan SX, Jiang DX, Hu RC, et al: Endoplasmic Reticulum Stress Induces HRD1 to Protect Alveolar Type II Epithelial Cells from Apoptosis Induced by Cigarette Smoke Extract. Cell Physiol Biochem 43:1337-1345, 2017

42. Tamargo-Gomez I, Marino G: AMPK: Regulation of Metabolic Dynamics in the Context of Autophagy. Int J Mol Sci 19, 2018

43. Liang Z, Yuan Z, Guo J, et al: Ganoderma lucidum Polysaccharides Prevent Palmitic Acid-Evoked Apoptosis and Autophagy in Intestinal Porcine Epithelial Cell Line via Restoration of Mitochondrial Function and Regulation of MAPK and AMPK/Akt/mTOR Signaling Pathway. Int J Mol Sci 20, 2019

44. Baskaran R, Poornima P, Priya LB, et al: Neferine prevents autophagy induced by hypoxia through activation of Akt/mTOR pathway and $\mathrm{Nrf2}$ in muscle cells. Biomed Pharmacother 83:1407-1413, 2016 


\section{Figures}

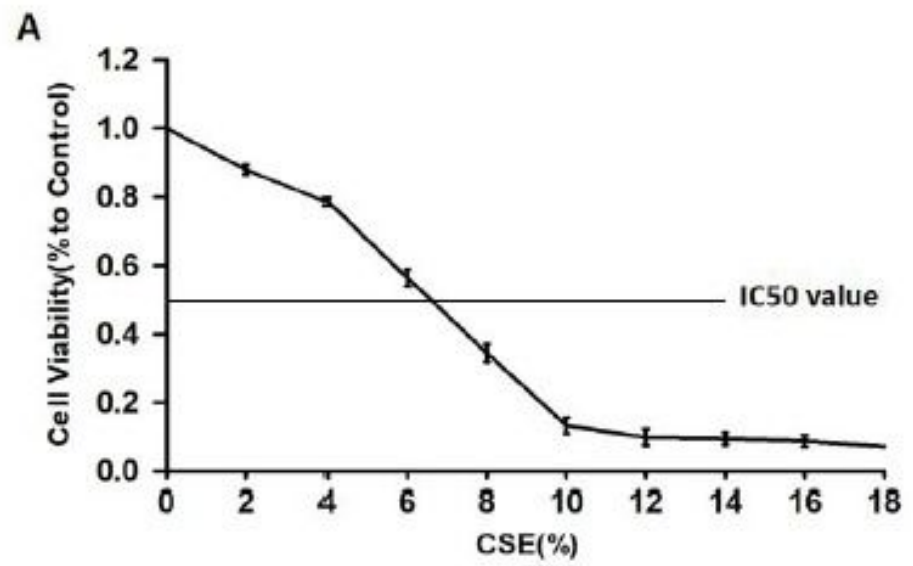

B
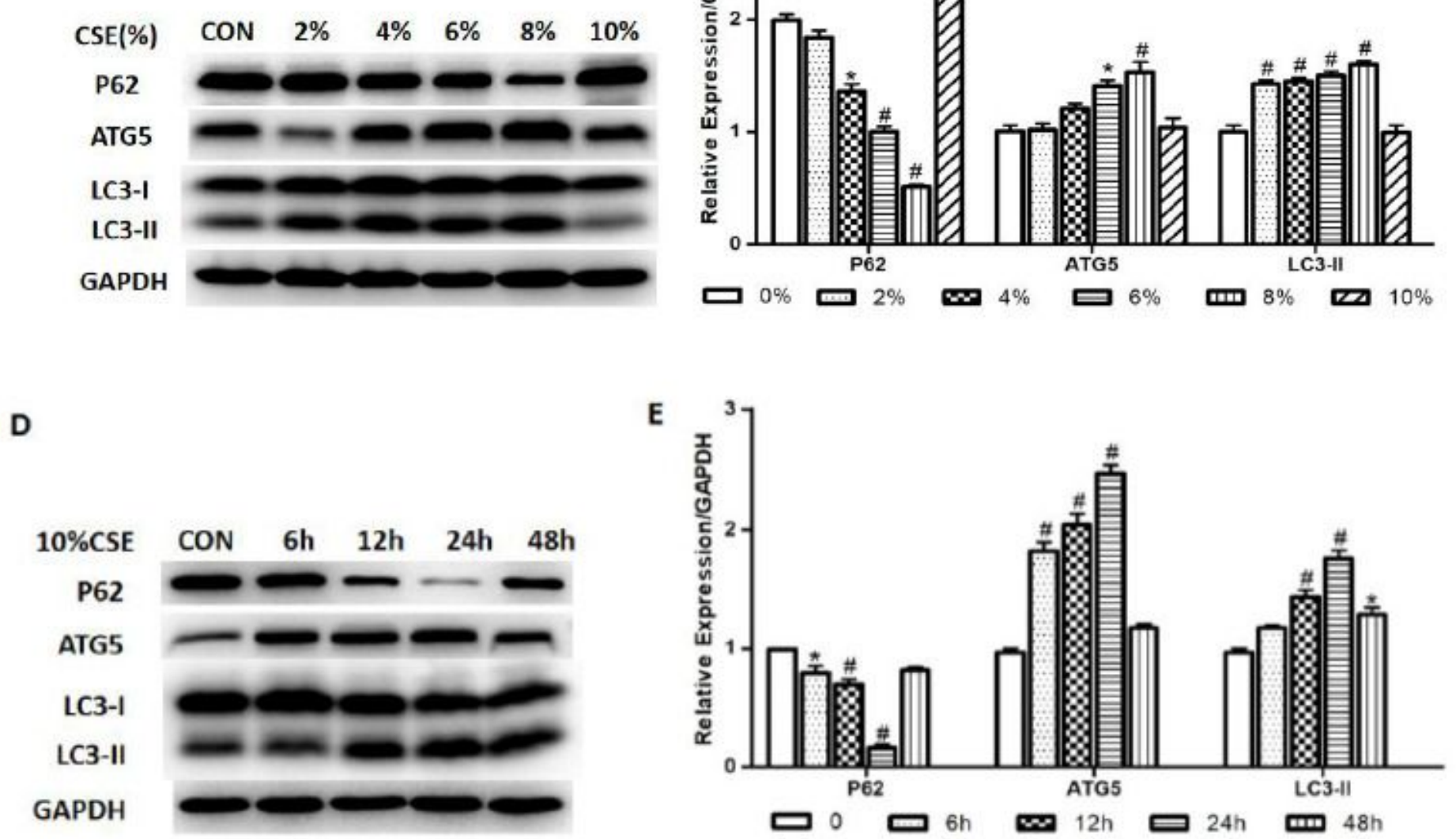

Figure 1

AEC-II was treated with CSE.(A) CCK8 showing various concentrations of CSE-induced changes in cell viability. Survival curve displayed IC50 value (6.8 \%). (B) GAPDH level is shown as a protein loading control. After 24h of various concentrations of CSE (0-10\%) treatment, P62, ATG5 and LC3 protein expression in AEC-II cells were determined by Western blotting analysis. (D) After being subjected to $10 \%$ of CSE for 0-48h. P62, ATG5 and LC3 protein expression were measured. (C, E) Western blotting analyses 
results showed average quantitative data and presented as the means \pm SD of at least 3 independent experiments. ${ }^{*} p<0.05$ compared with control; $\# p<0.01$ compared with control.

A

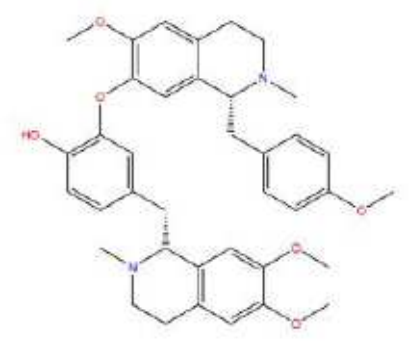

Neferine Chemical Structure

C

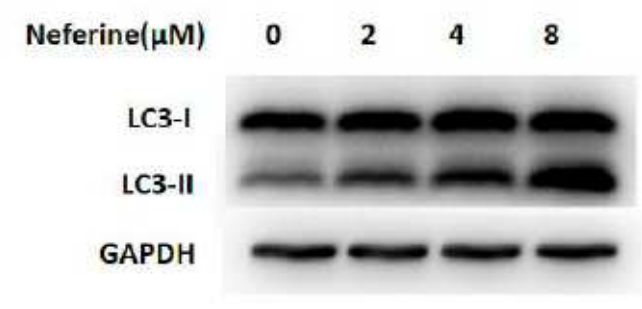

B

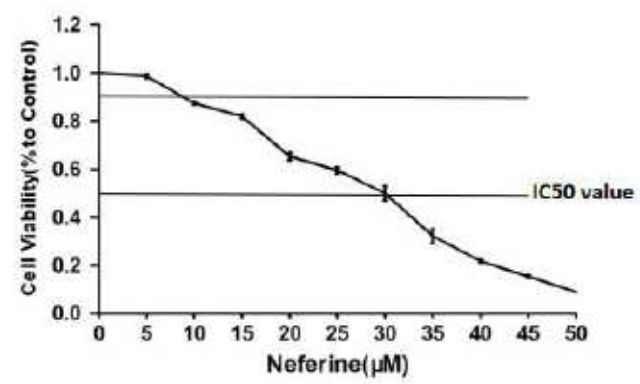

D

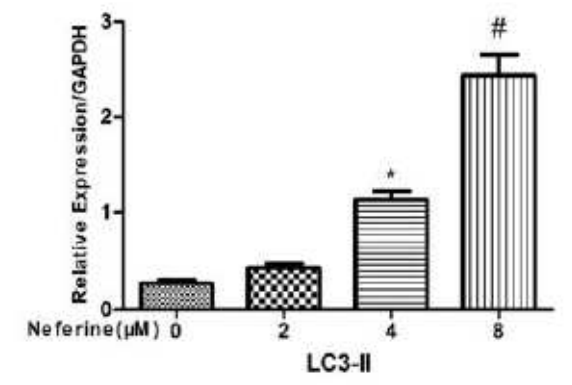

$\mathbf{E}$
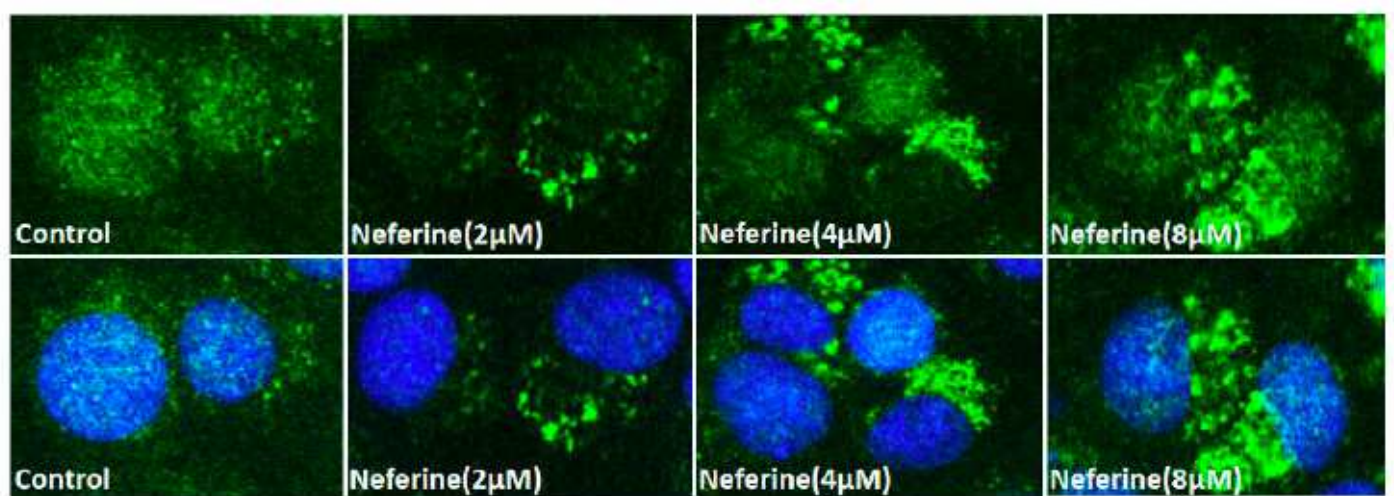

F

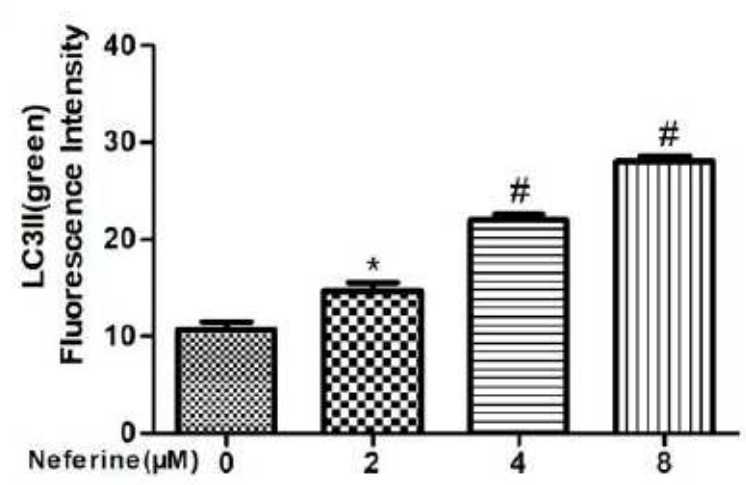

Figure 2

AEC-II was treated with neferine. (A) The chemical structure of neferine . (B) CCK8 showing various concentrations of neferine-induced changes after $24 \mathrm{~h}$ in cell viability. Survival curve displayed IC50 value $(30 \mu \mathrm{M})$. (C) GAPDH level is shown as a protein loading control. After $24 \mathrm{~h}$ for different concentrations (2, 
4, and $8 \mu \mathrm{M}$ ), LC3 protein expression in AEC-II cells were determined by Western blotting analysis. (E) Treated with neferine, IF images of AEC-II induced LC3II expression changes (green). Nuclei was labeled with DAPI (blue). (D,F) Results are presented as the means \pm SD of at least 3 independent experiments. * $\mathrm{p}<0.05$ compared to control; \# $\mathrm{p}<0.01$ compared to control.

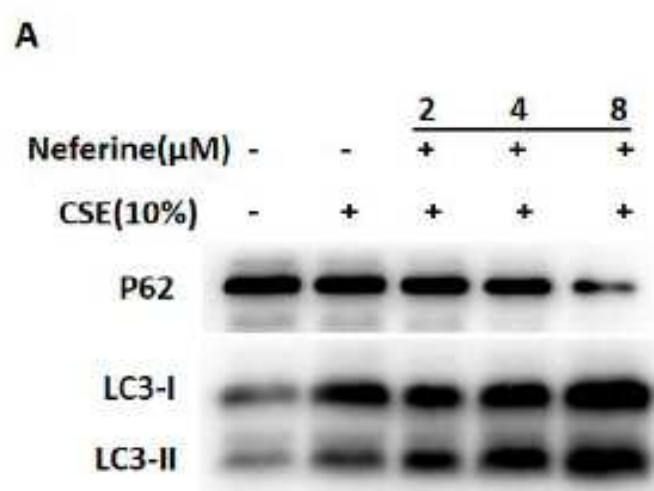

GAPDH
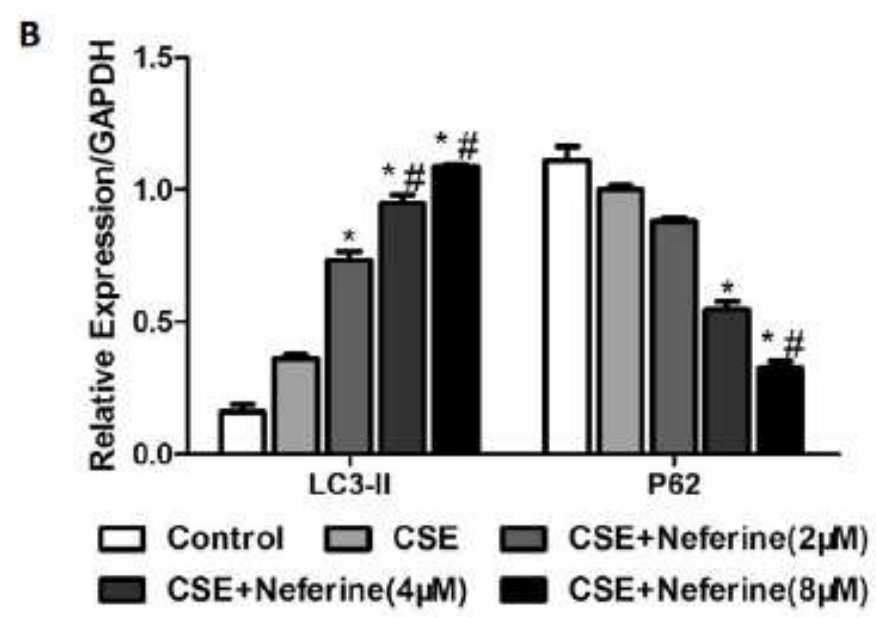

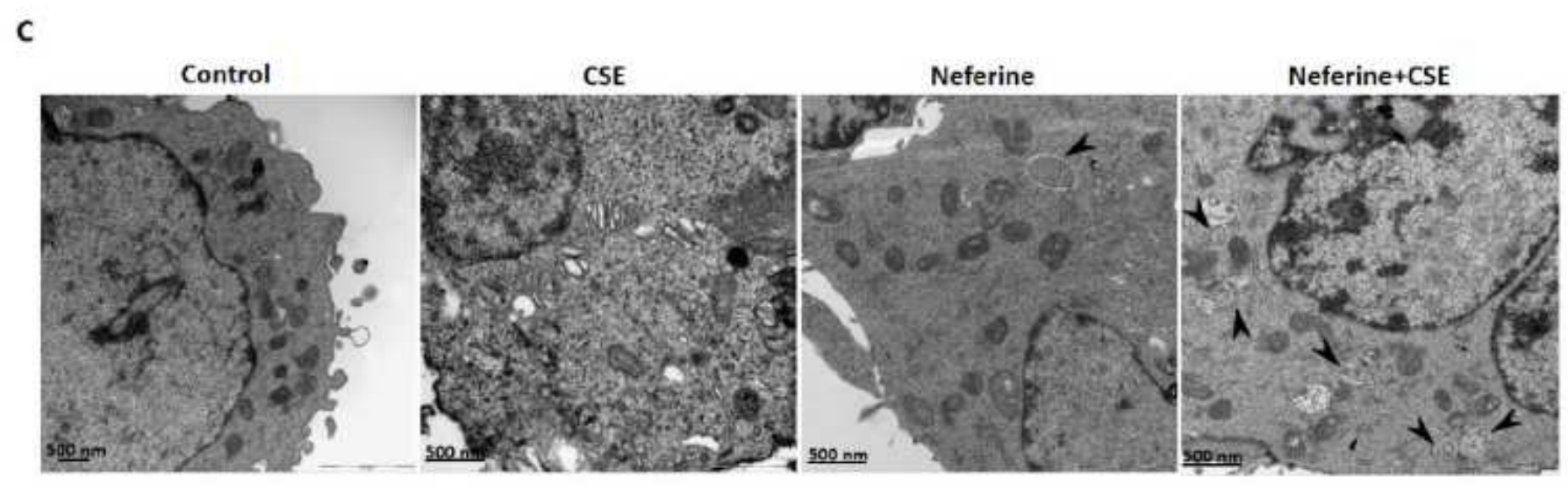

\section{Figure 3}

Effect of neferine on AEC-II survival in CSE by autophagy. (A,B) GAPDH level is shown as a protein loading control. After $2 \mathrm{~h}$ for different concentrations neferine $(0,2,4$,and $8 \mu \mathrm{M})$ with/without $10 \% \mathrm{CSE}$ for 24h, LC3 and P62 protein expression in AEC-II cells were determined by Western blotting analysis. (C) After $2 \mathrm{~h}$ for neferine at a dose of $8 \mu \mathrm{M}$ with/without $10 \% \mathrm{CSE}$ for $24 \mathrm{~h}$, electron microscope images presented representative autophagosomes or autolysosomes (marked by black arrows;Bar, $500 \mathrm{~nm}$ ). Results are presented as the means \pm SD of at least 3 independent experiments. ${ }^{*} p<0.05$ compared to control; $\# \mathrm{p}<0.05$ compared to the CSE group without neferine. 

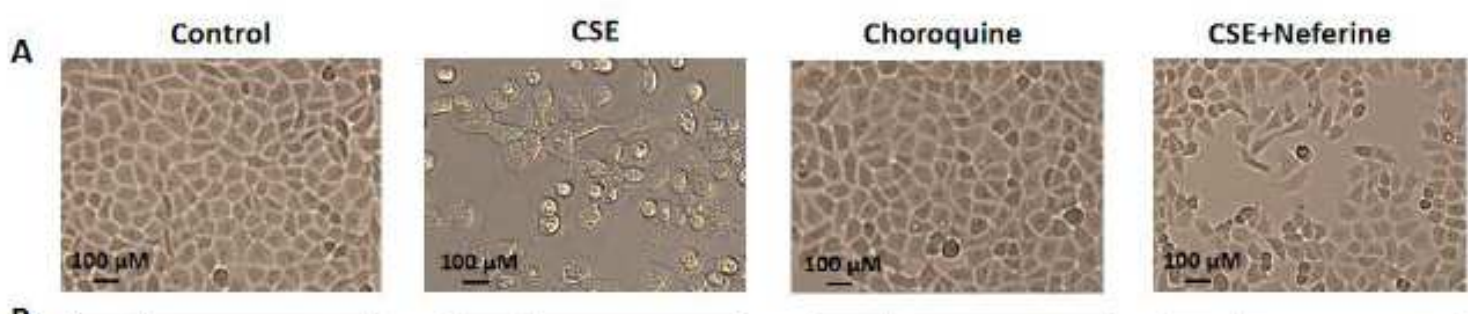

CSE + Neferine + Choroquine

B
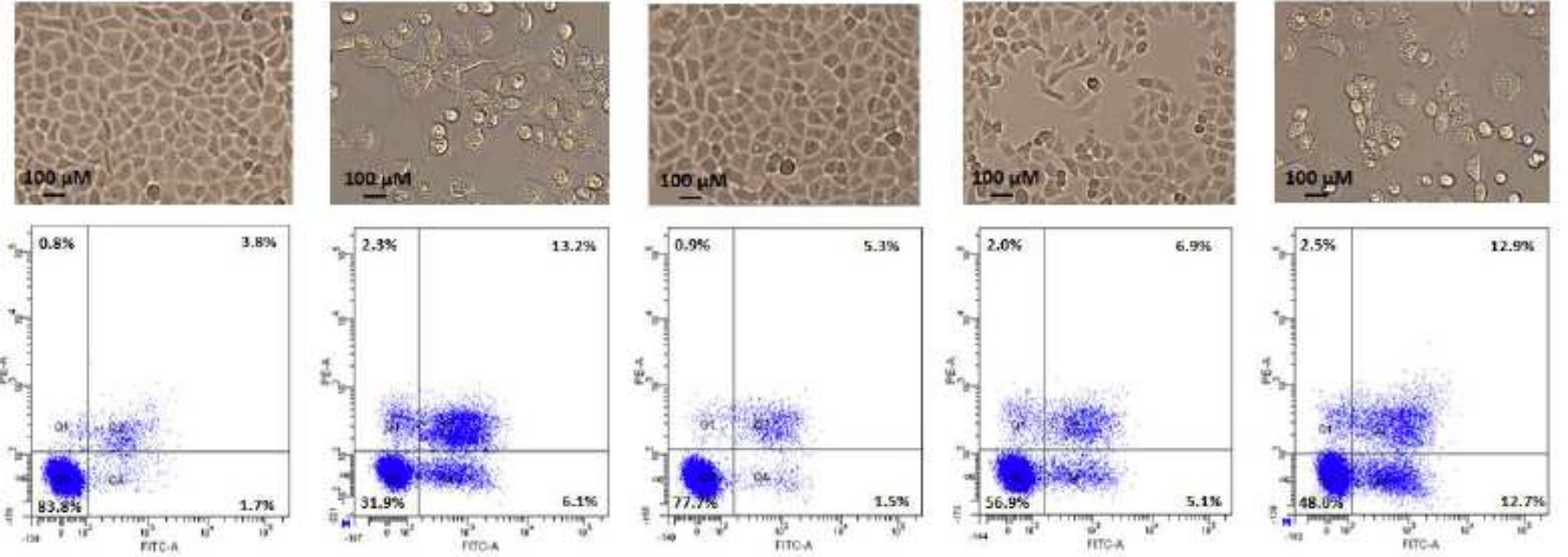

C
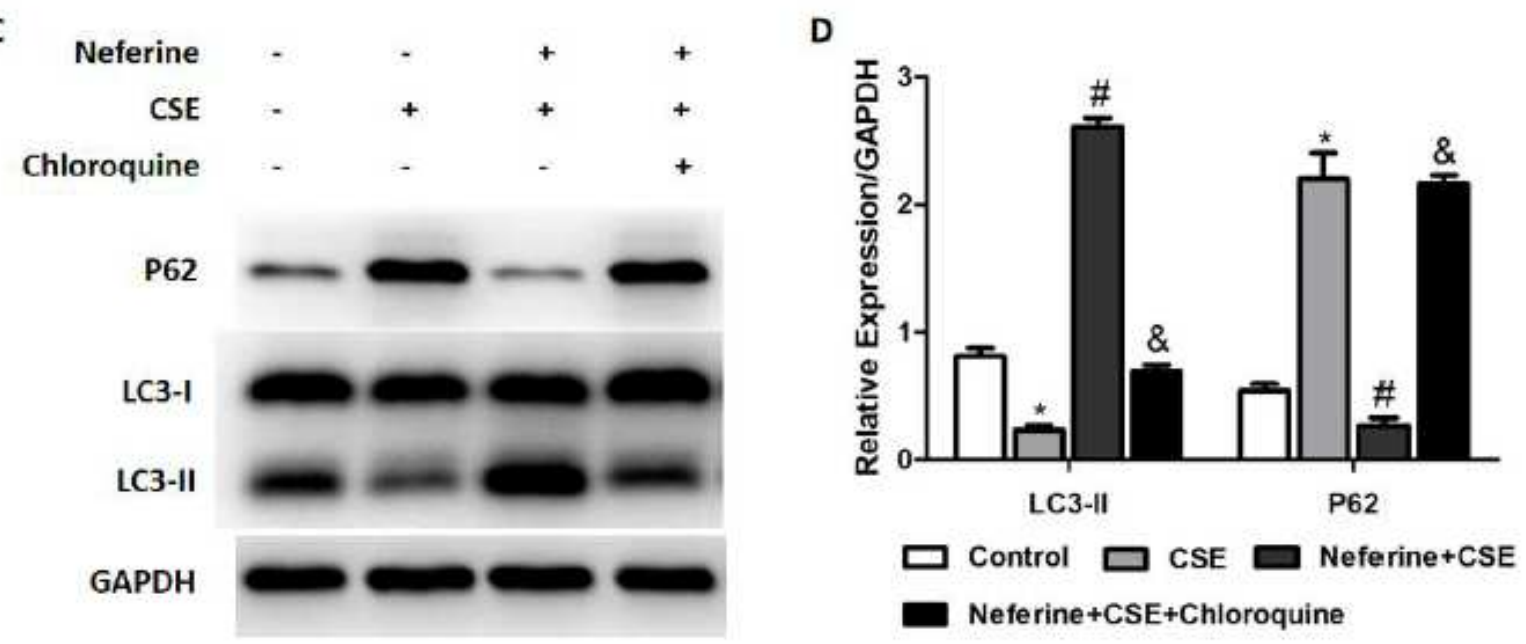

\section{Figure 4}

Autophagy inhibitor attenuated neferine's effect in protecting AEC-II against CSE. AEC-II were pretreated for $2 \mathrm{~h}$ with neferine $(8 \mu \mathrm{M})$, or neferine $(8 \mu \mathrm{M})$ together with chloroquine $(20 \mu \mathrm{M})$, followed by treatment with $10 \% \mathrm{CSE}$ for $24 \mathrm{~h}$. (A) Phase-contrast microscopy images presented cells viability (Bar, $100 \mu \mathrm{m})$. (B) AEC-II was analyzed by flow cytometric analysis of apoptosis. $(C, D)$ GAPDH level is shown as a protein loading control. LC3 and P62 protein expression in AEC-II were determined by Western blotting analysis. Results are presented as the means \pm SD of at least 3 independent experiments. ${ }^{*} p<0.05$ compared to control; \# $p<0.05$ compared to the CSE group; \& $p<0.05$ compared to the CSE and neferine group. 

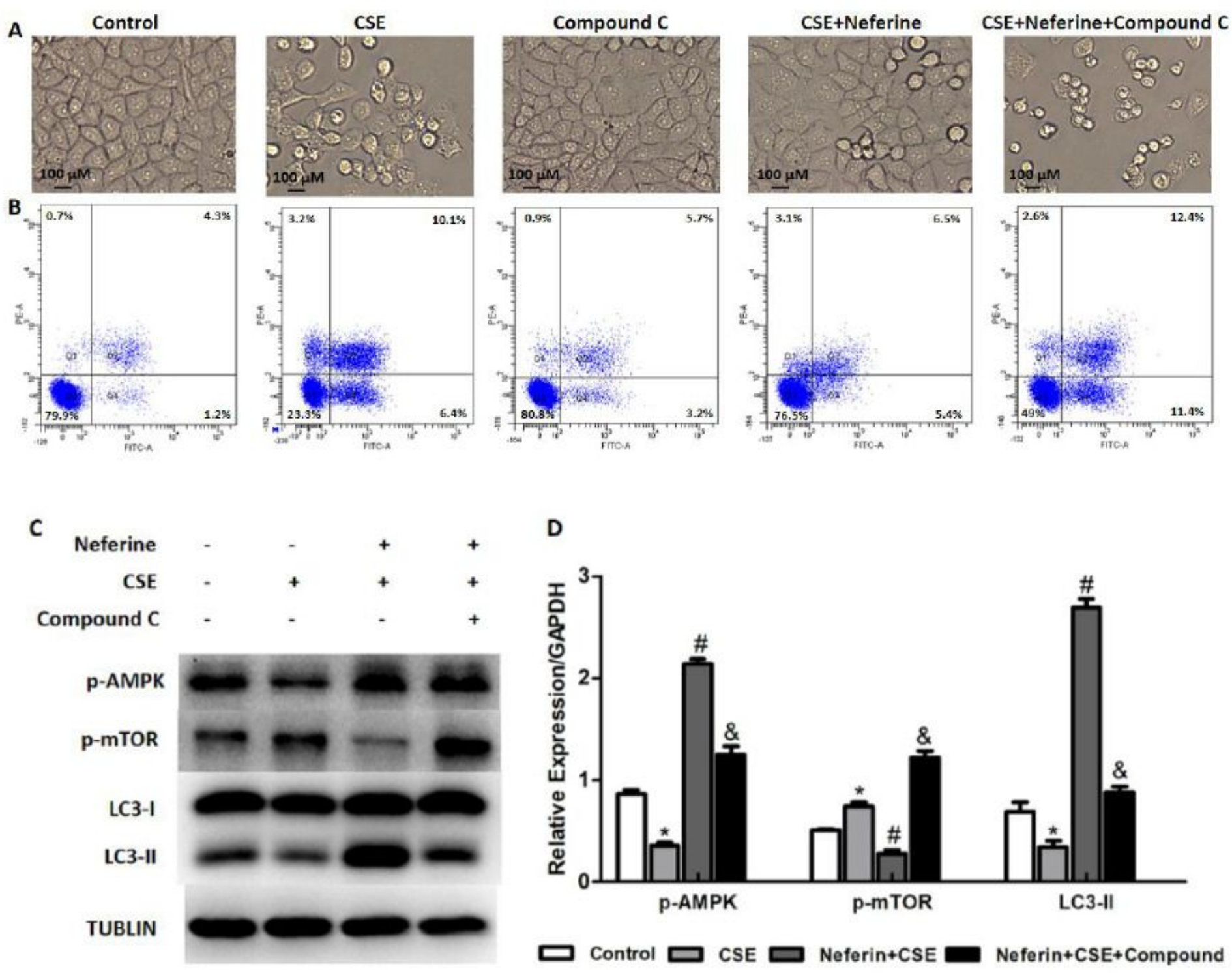

\section{Figure 5}

Neferine protected AEC-II against CSE via the AMPK/mTOR pathway. AEC-II were pretreated for $2 \mathrm{~h}$ with neferine $(8 \mu \mathrm{M})$, or neferine $(8 \mu \mathrm{M})$ together with Compound $\mathrm{C}(10 \mu \mathrm{M})$, followed by treatment with $10 \%$ CSE for 24h. (A) Phase-contrast microscopy images presented cells viability (Bar, $100 \mu \mathrm{m}$ ). (B) AEC-II was analyzed by flow cytometric analysis of apoptosis. $(C, D)$ GAPDH level is shown as a protein loading control. LC3, p-AMPK, and p-mTOR protein expression in AEC-II were determined by Western blotting analysis. Results are presented as the means \pm SD of at least 3 independent experiments. * $p<0.05$ compared to control; \# $p<0.05$ compared to the CSE group; \& $p<0.05$ compared to the CSE and neferine group. 